ACTA ARITHMETICA

XCVIII.4 (2001)

\title{
On some sums involving the largest prime divisor of $n$, II
}

\author{
by \\ E. J. Scourfield (London)
}

In memory of Paul Erdös

1. Introduction. Several authors have considered (in [3], [5]-[7], [9], for example) sums of one of the types

$$
\Sigma_{f}(x)=\sum_{\substack{n f(P(n)) \leq x \\ n>1}} 1, \quad \Sigma_{f}^{\prime}(x)=\sum_{1<n \leq x} \frac{1}{f(P(n))}
$$

for certain special functions $f$, such as $f(w)=w^{\gamma}$ or $(\log w)^{\gamma}$ with $\gamma>0$, where

$$
P(1)=1, \quad P(n)=\max _{p \mid n} p \quad \text { for } n>1 \quad(p \text { prime }) .
$$

Their aim was to find the order of magnitude of the sum or the main term in its asymptotic formula. However the error term obtained usually depended on that in Lemma 2.5 below and so was rather weak. The results below show that in fact the magnitude of their error terms is best possible (see Corollaries 1 and 2 in Section 4).

The motivation for writing this paper is twofold. There are many standard functions $f(w)$ between and beyond powers of $w$ or of $\log w$, and it is interesting to discover how the magnitude of the sums in (1.1) changes as the size of $f(w)$ increases. This is illustrated in the example given after the statement of Theorem 1 in this section. For a general function of the type considered in the present paper this question is answered in Lemma 3.4 together with equation (1.12) and Lemma 3.7. To establish these results, we pick out those $n$ contributing the bulk of this magnitude, and this turns out to be the integers $n$ whose largest prime factor lies in a certain range.

Our second objective is to obtain an error term that is much smaller than the error terms of previous investigations. This is accomplished by finding explicitly a second term in the asymptotic formulae for the sums

2000 Mathematics Subject Classification: Primary 11N37. 
(1.1) which, like the main term, involves the extensively studied Dickman function $\varrho(u)$, defined in (1.15). We establish our main results both unconditionally and on the assumption of the Riemann Hypothesis. We concentrate on the slightly more complicated sum $\Sigma_{f}(x)$; the corresponding formulae for $\Sigma_{f}^{\prime}(x)$ then follow by a similar argument. Our proof depends on using a result (Lemma 2.7) due to E. Saias [8] instead of the less precise formula in Lemma 2.5.

We begin by describing the class of functions $f$ considered, which includes the special cases quoted above. The idea is to consider unbounded functions $f(w)$ such that $\log f(w)$ is approximately a fixed power of $\log w$ so that $f(w)$ is not too large. Throughout this paper we assume the following

Conditions on $f$.

(i) $f(w)$ is positive and strictly increasing on $w \geq 1$ with a continuous derivative on $w \geq w_{0}$ for some fixed $w_{0} \geq 1$;

(ii) there exists a fixed $\nu \geq 0$ such that

$$
\log f(w)=(\log w)^{\nu} \eta(w)
$$

where

$$
\eta(w)=(\log w)^{\delta(w)} \quad \text { with } \quad \delta(w)=o(1) \text { as } w \rightarrow \infty,
$$

$\eta(w)$ is monotonic, and, when $\nu=0, \eta(w) \rightarrow \infty$ as $w \rightarrow \infty$;

(iii) we have

$$
\xi(w):=\frac{\eta^{\prime}(w)}{\eta(w)} w \log w=o(1) \quad \text { as } w \rightarrow \infty ;
$$

(iv) when $\nu>0$, for any positive bounded $d$ with $d \gg 1$,

$$
\eta\left(w^{d}\right)=\eta(w)(1+O(|\xi(w)|)) .
$$

It follows from (1.5) that when $\nu>0$,

$$
\log f\left(w^{d}\right)=\left(d^{\nu}+O(|\xi(w)|)\right) \log f(w)
$$

for positive $d$ with $1 \ll d \ll 1$.

Conditions (iii) and (iv) hold for the "obvious" choices for $\eta(w)$ in (1.2); we illustrate this with the following

ExAmples. Let $\gamma>0$ and $\lambda \neq 0$ be constants with $\lambda>0$ when $\nu=0$ but $\lambda$ of either sign otherwise.

(1) $\log f(w)=\gamma(\log w)^{\nu}$, so $\eta(w)=\gamma, \delta(w)=0, \xi(w)=0$ and, by (i) and (ii), $\nu>0$. When $\nu=1, f(w)=w^{\gamma}$.

(2) $\log f(w)=(\log w)^{\nu}\left(\log _{2} w\right)^{\lambda}$, so

$$
\eta(w)=\left(\log _{2} w\right)^{\lambda}, \quad \delta(w)=\frac{\lambda \log _{3} w}{\log _{2} w}, \quad \xi(w)=\frac{\lambda}{\log _{2} w}
$$


where $\log _{2} w=\log (\log w)$ for $w>1, \log _{3} w=\log \left(\log _{2} w\right)$ for $w>e$. When $\nu=0$ and $\lambda=1, f(w)=\log w$.

Combining examples (1) and (2), $f(w)=(\log w)^{\gamma}$ when $\nu=0$ and $\eta(w)=\gamma \log _{2} w$.

(3) $\log f(w)=(\log w)^{\nu} \exp \left(\left(\log _{3} w\right)^{\lambda}\right)=\exp \left(\nu \log _{2} w+\left(\log _{3} w\right)^{\lambda}\right)$, so

$$
\eta(w)=\exp \left(\left(\log _{3} w\right)^{\lambda}\right), \quad \delta(w)=\frac{\left(\log _{3} w\right)^{\lambda}}{\log _{2} w}, \quad \xi(w)=\frac{\lambda\left(\log _{3} w\right)^{\lambda-1}}{\log _{2} w} .
$$

When $\lambda=1$, we have $\eta(w)=\log _{2} w$, which is included in (2).

(4) $\log f(w)=(\log w)^{\nu} \exp \left(\left(\log _{2} w\right)^{\lambda}\right)=\exp \left(\nu \log _{2} w+\left(\log _{2} w\right)^{\lambda}\right)$, with $\lambda<1$ (in order that (1.3) is satisfied), so

$$
\eta(w)=\exp \left(\left(\log _{2} w\right)^{\lambda}\right), \quad \delta(w)=\left(\log _{2} w\right)^{\lambda-1}, \quad \xi(w)=\lambda \delta(w) .
$$

When $\lambda$ is near 1 , the functions of the form $\eta(w)$ in (4) are among the largest permitted.

In examples (2)-(4), $(\log w)^{-\nu} \log f(w)$ is strictly monotonic when $\lambda \neq 0$.

It is interesting to compare the sums $\Sigma_{f}(x), \Sigma_{f}^{\prime}(x)$ for the class of functions $f$ described above. In [10] we obtained the rather surprising result that for $f$ satisfying conditions (i) and (ii) above,

$$
\Sigma_{f}(x) \sim \Sigma_{f}^{\prime}(x) \quad \text { as } x \rightarrow \infty
$$

if and only if

$$
\frac{\log f(w)}{\log w} \log _{2} w=o(1) \quad \text { as } w \rightarrow \infty .
$$

Thus (1.7) holds when $\nu<1$ or $\nu=1$ and $\eta(w) \log _{2} w=o(1)$. We were actually able to consider a wider class of functions in that paper, but here we require stronger conditions on $f$ in order to obtain good error terms.

Before stating our main result, we need some further definitions. Assume throughout that $x$ is sufficiently large and that $\varepsilon>0$ is fixed but sufficiently small. The iterative logarithms are defined in example (2) above.

Definitions. Define $X=X(x)$ and $W=W(x)$ by

$$
X f(X)=x, \quad W^{2} f(W)=x .
$$

When $\nu>0$, define $L=L(x)$ by

$$
(1+\nu) \log L \log f(L)=\log x \log _{2} x .
$$

When $0 \leq \nu<3 / 5$, define $M=M(x)$ by

$$
(8 / 5-\varepsilon)(\log M)^{8 / 5-\varepsilon}=\log x \log _{2} x
$$

(where $\varepsilon<3 / 5-\nu$ ). 
We note that

$$
\begin{gathered}
\log L=\left(\frac{\log x \log _{2} x}{1+\nu}\right)^{1 /(1+\nu)+o(1)}, \\
\log f(L)=\left(\frac{\log x \log _{2} x}{1+\nu}\right)^{\nu /(1+\nu)+o(1)}, \\
\frac{\log x \log _{2} x}{\log M}=\left(\frac{8}{5}-\varepsilon\right)^{5 /(8-5 \varepsilon)}\left(\log x \log _{2} x\right)^{(3-5 \varepsilon) /(8-5 \varepsilon) .}
\end{gathered}
$$

We observe that when $0<\nu<3 / 5$,

$$
M<L \quad \text { and } \quad \log f(L)<\frac{\log x \log _{2} x}{\log M}
$$

provided $\varepsilon$ is sufficiently small and $x$ sufficiently large.

The well known Dickman function $\varrho(u)$ is defined by the differentialdifference equation

$$
\begin{cases}\varrho(u)=0 & \text { for } u<0 \\ \varrho(u)=1 & \text { for } 0 \leq u \leq 1 \\ u \varrho^{\prime}(u)+\varrho(u-1)=0 & \text { for } u>1\end{cases}
$$

Let

$$
\begin{gathered}
u(x, w)=\frac{\log x-\log f(w)}{\log w}-1 \\
H(x)=\int_{2}^{x} \frac{1}{w f(w) \log w} \\
\quad \times\left\{\varrho(u(x, w))-\int_{1}^{x} \frac{t-[t]}{t^{2} \log w} \varrho^{\prime}\left(u\left(\frac{x}{t}, w\right)\right) d t\right\} d w \\
H_{0}(x)=\int_{W}^{X}\left(\frac{x}{w f(w)}-\left[\frac{x}{w f(w)}\right]\right) \frac{1}{\log w} d w .
\end{gathered}
$$

Note that $\varrho(u(x, w))=0$ when $w>X$ and $\varrho^{\prime}(u(x / t, w))=0$ when $w^{2} f(w)>$ $x / t, w f(w) \neq x / t$.

Our main aim in this paper is to prove

THEOREM 1. Let $\varepsilon>0$ be sufficiently small and $L, M$ be defined by (1.9), (1.10) respectively.

(i) When $\nu \geq 3 / 5$,

$$
\Sigma_{f}(x)=x H(x)+O\left(x H(x) \exp \left(-(\log L)^{3 / 5-\varepsilon}\right)\right) .
$$


(ii) When $3 / 8 \leq \nu<3 / 5$,

$$
\Sigma_{f}(x)=x H(x)+O\left(x \exp \left(-(1+o(1)) \frac{\log x \log _{2} x}{\log M}\right)\right) .
$$

(iii) When $0 \leq \nu<3 / 8$,

$$
\Sigma_{f}=x H(x)-H_{0}(x)+O\left(x \exp \left(-(1+o(1)) \frac{\log x \log _{2} x}{\log M}\right)\right) .
$$

We remark that the $O$-terms in (ii) and (iii) are indeed error terms by Lemmas 3.4 and 3.7 and (1.14). To give the reader some idea of their size compared to that of the main term in Theorem 1, we look at the basic functions $f(w)$ with $\eta(w)=1$.

EXAMPLE. Let $f(w)=\exp \left((\log w)^{\nu}\right)$ with $\nu>0$. The magnitude of the main term $x H(x)$ in Theorem 1 is

$$
x \exp \left(-(1+\nu+o(1))\left(\frac{\log x \log _{2} x}{1+\nu}\right)^{\nu /(1+\nu)}\right)
$$

whenever $\nu>0$ (see Lemma 3.4). The form of an estimate for $\Sigma_{f}(x)-x H(x)$ depends on the size of $\nu$, for the dominant error term changes.

(i) $\nu \geq 3 / 5$. The error term is obtained by multiplying the main term $x H(x)$ by

$$
\exp \left(-(\log L)^{3 / 5-\varepsilon}\right)=\exp \left(-\left(\frac{\log x \log _{2} x}{1+\nu}\right)^{(3-5 \varepsilon) /(5(1+\nu))}\right)
$$

by (1.9).

(ii) $0<\nu<3 / 5$. From (1.13), the $O$-term is

$$
x \exp \left(-(c+o(1))\left(\log x \log _{2} x\right)^{3 / 8-\varepsilon_{1}}\right)
$$

where $\varepsilon_{1}=25 \varepsilon /(8(8-5 \varepsilon))$ and $c=(8 / 5-\varepsilon)^{5 /(8-5 \varepsilon)}$.

Note that $0<\nu /(1+\nu)<3 / 8$ if and only if $0<\nu<3 / 5$, so that this $O$-term is smaller than $x H(x)$. However this $O$-term is less than that in (i) above when $\nu \geq 3 / 5$.

(iii) $0<\nu<3 / 8$. In this case $0<\nu /(1+\nu)<3 / 11<3 / 8$ and the $O$-term is as in (ii). Consider the extra main term $H_{0}(x)$ defined in (1.18) where $W, X$ are given in (1.8). On using the substitution $v=x /(w f(w))$ and observing that

$$
\frac{-x}{v^{2}} \frac{d v}{d w}=\left(1+\nu(\log w)^{\nu-1}\right) \exp \left((\log w)^{\nu}\right)
$$

and that $v=1$ when $w=X, v=W$ when $w=W$, we find by a simple calculation that 


$$
\begin{aligned}
&(C+o(1)) \frac{x}{\log x} \exp (-\left.(1+o(1))(\log x)^{\nu}\right) \leq H_{0}(x) \\
& \leq(2 C+o(1)) \frac{x}{\log x} \exp \left(-(1+o(1))\left(\frac{1}{2} \log x\right)^{\nu}\right)
\end{aligned}
$$

where

$$
C=\int_{1}^{\infty} \frac{v-[v]}{v^{2}} d v
$$

Thus $H_{0}(x)=o(x H(x))$ since $\nu>\nu /(1+\nu)$ but $H_{0}(x)$ exceeds the error term in (ii) since $\nu<3 / 8$.

Next we state the corresponding result when the Riemann Hypothesis is assumed. The shape of the results is similar but the boundary values for $\nu$ in the three cases increase from $3 / 8,3 / 5$ to $1 / 2,1$ respectively, and the error terms are stronger (for compare (1.13) with (1.20)). We replace $M$ by a quantity $N$ given by the

DEFinition. When

$$
\lim _{w \rightarrow \infty} \frac{\log f(w)}{\log w}=k \quad \text { with } 0 \leq k<\infty,
$$

define $N=N(x)$ by

$$
\log N=\left(\frac{\log x \log _{2} x}{2 k+1-2 \varepsilon}\right)^{1 / 2}
$$

where $\varepsilon$ is sufficiently small and in particular $0<\varepsilon<1 / 2$.

Note that

$$
\frac{\log x \log _{2} x}{\log N}=\left((2 k+1-2 \varepsilon) \log x \log _{2} x\right)^{1 / 2}
$$

and, since $\nu<1$ or $\nu=1$ and $\lim _{w \rightarrow \infty} \eta(w)=k$, for $\nu>0$ we have

$$
N<L \quad \text { and } \quad \log f(L)<\frac{\log x \log _{2} x}{\log N} .
$$

Moreover, when $N$ is defined, $\log f(W) \geq\left(\log x \log _{2} x\right) /(\log N)$ when $\nu>1 / 2$ and for some $f$ when $\nu=1 / 2$ but not otherwise.

THEOREM 2. Assume the Riemann Hypothesis. Let $\varepsilon>0$ be sufficiently small.

(i) When $\frac{\log f(w)}{\log w} \rightarrow \infty$ as $w \rightarrow \infty$,

$$
\Sigma_{f}(x)=x\left(1+O\left(L^{-1 / 2+\varepsilon}\right)\right) H(x) .
$$


(ii) When $\lim _{w \rightarrow \infty} \frac{\log f(w)}{\log w}$ is finite and $\log f(W) \geq \frac{\log x \log _{2} x}{\log N}$,

$$
\Sigma_{f}=x H(x)+O\left(x \exp \left(-(1+o(1)) \frac{\log x \log _{2} x}{\log N}\right)\right) .
$$

(iii) When $\lim _{w \rightarrow \infty} \frac{\log f(w)}{\log w}=0$ and $\log f(W)<\frac{\log x \log _{2} x}{\log N}$,

$$
\Sigma_{f}(x)=x H(x)-H_{0}(x)+O\left(x \exp \left(-(1+o(1)) \frac{\log x \log _{2} x}{\log N}\right)\right) .
$$

As in Theorem 1, (1.21) implies that the $O$-terms in (ii) and (iii) are error terms.

For completeness, we state the corresponding results for the second sum $\Sigma_{f}^{\prime}(x)$ in (1.1). Corresponding to $H(x), H_{0}(x)$, we define

$$
\begin{aligned}
H^{\prime}(x)= & \int_{2}^{x} \frac{1}{w f(w) \log w}\left\{\varrho\left(\frac{\log x}{\log w}-1\right)\right. \\
& \left.-\int_{1}^{x} \frac{t-[t]}{t^{2} \log w} \varrho^{\prime}\left(\frac{\log (x / t)}{\log w}-1\right) d t\right\} d w, \\
H_{0}^{\prime}(x)= & \int_{\sqrt{x}}^{x}\left(\frac{x}{w}-\left[\frac{x}{w}\right]\right) \frac{1}{f(w) \log w} d w .
\end{aligned}
$$

TheOREm 3. In the statements of Theorems 1 and 2 replace $\Sigma_{f}(x)$, $H(x), H_{0}(x)$ by $\Sigma_{f}^{\prime}(x), H^{\prime}(x), H_{0}^{\prime}(x)$ respectively. Then the corresponding results hold.

It may be helpful to the reader to outline the main steps in the proofs of these theorems, which depend on expressing the sums $\Sigma_{f}(x), \Sigma_{f}^{\prime}(x)$ in terms of the well known function

$$
\Psi(x, y)=\#\{n \leq x: P(n) \leq y\},
$$

investigated by de Bruijn. We use the standard approximation for it given in Lemma 2.5 and a more precise result given in Lemma 2.7. We see from (3.1) that

$$
\Sigma_{f}(x)=\sum_{p f(p) \leq x} \Psi\left(\frac{x}{p f(p)}, p\right) .
$$

Our first aim, accomplished in Section 3, is to determine the order of magnitude of the sum on the right by first identifying the dominant range for $p$. When $\nu>0$ this turns out to be a range containing the quantity $L$ given by (1.9) (see Lemmas 3.3, 3.4 in which an estimate for $\Sigma_{f}(x)$ is obtained). In Lemma 3.5 a weak asymptotic formula for $\Sigma_{f}(x)$ is then derived. The case 
$\nu=0$ is investigated at the end of Section 3 , but here the dominant range for $p$ in the sum (1.23) contains the largest primes $p$ satisfying $p f(p) \leq x$.

The goal in Section 4 is to replace the asymptotic formula in Lemma 3.5 (for $\nu>0$ ) or Lemma 3.7(ii) (for $\nu=0$ ) by one with further terms and a much stronger error term. This is achieved by using the formula for $\Psi(x, y)$ in Lemma 2.7 instead of that in Lemma 2.5 and applying partial summation to obtain in Lemma 4.1 a more precise formula for the dominant part of the sum in (1.23). We find we need to distinguish between the ranges $\nu \geq 3 / 5$, $3 / 8 \leq \nu<3 / 5$ and $0 \leq \nu<3 / 8$, which lead to the three cases of Theorem 1 , for in these three ranges different error terms dominate, as they did in the example above. In addition, when $0 \leq \nu<3 / 8$ there is a secondary main term $H_{2}(x)$ in Lemma 4.1 that measures the contribution from the largest primes in the sum (1.23). Theorem 1 then follows.

In Section 5 we assume the Riemann Hypothesis and indicate how Theorem 2 is proved by the method used to establish Theorem 1. The corresponding results for the sum $\Sigma_{f}^{\prime}(x)$ in (1.1), stated in Theorem 3, can be derived by similar methods (see the end of Section 4 ).

Subsidiary results required in later sections are given in Section 2.

The author thanks the referee for his helpful suggestions concerning the presentation of this paper.

\section{Preliminary lemmas}

LEMMA 2.1.

$$
\frac{f^{\prime}(w)}{f(w)}=\frac{\log f(w)}{w \log w}(\nu+\xi(w)) .
$$

This follows from conditions (ii) and (iii) on $f$.

LEMMA 2.2. The function $\varrho(u)$ defined in (1.15) has the following properties:

(i) As $u \rightarrow \infty$

$$
\varrho(u)=\exp \left(-u\left(\log u+\log _{2} u-1+O\left(\frac{\log _{2} u}{\log u}\right)\right)\right) .
$$

(ii) $\varrho(u)$ is continuous except at $u=0$;

$\varrho^{\prime}(u)$ is defined for $u \neq 0$ and continuous except at $u=1$;

$0<\varrho(u) \leq 1$ for $u \geq 0$ and $-1 \leq \varrho^{\prime}(u)<0$ for $u>1$;

$\varrho(u)$ decreases strictly and $\varrho^{\prime}(u)$ increases strictly on $u>1$.

(iii) (a) $\varrho^{\prime}(u)=-\varrho(u)\left(\log u+O\left(\log _{2} u\right)\right)$ for $u \geq 3$.

(b) $\varrho(u-v)=\varrho(u) \exp \left(v\left(\log u+O\left(\log _{2} u\right)\right)\right)$ for $|v| \leq 2 u / 3$, $u-v \geq 3, u \geq 3$. 
Proof. A stronger form of (i) is due to de Bruijn [2], (ii) follows from the definition (1.15) of $\varrho(u)$, and (iii)(a) is the case $k=1$ of Lemma 3(viii) of [8]. Part (b) of (iii) follows from (a) on noting that

$$
\begin{aligned}
\log \frac{\varrho(u-v)}{\varrho(u)} & =\int_{u-v}^{u}-\frac{\varrho^{\prime}(t)}{\varrho(t)} d t=\int_{u-v}^{u}\left(\log t+O\left(\log _{2} t\right)\right) d t \\
& =v\left(\log u+O\left(\log _{2} u\right)\right)
\end{aligned}
$$

under the conditions stated.

Definitions. Define $Z=Z(x)$ by

$$
\log Z=\left(\log _{2} x\right)^{2} .
$$

When $\nu \geq 3 / 5$, define $\alpha, L_{2}, Y$ by

$$
\alpha^{\nu}=\frac{3}{2}(1+\nu), \quad L_{2}=L^{\alpha}, \quad Y=f\left(L_{2}\right),
$$

where $L$ is given by (1.9).

When $0 \leq \nu<3 / 5$, define $Y$ and $L_{2}$ by

$$
\log Y=\frac{\log x \log _{2} x}{\log M}, \quad f\left(L_{2}\right)=\min (Y, f(X)),
$$

where $M$ is given by (1.10).

We observe that $L_{2}=X$ in (2.3) if and only if $0 \leq \nu<3 / 8$.

Let $K(x, w)$ denote a function (not always the same one) satisfying

$$
K(x, w)=\log \left(\frac{\log x}{\log w}\right)+O\left(\log _{2}\left(\frac{\log x}{\log w}\right)\right) .
$$

We defined $u(x, w)$ in (1.16).

LEMMA 2.3. For $Z \leq w \leq \min \left(L_{2}, x^{1 / 5}\right)$ and $1 \leq t \leq Y$,

$$
\varrho^{\prime}(u(x / t, w))=-K(x, w) \varrho(u(x, w)) \exp \left(\frac{\log t}{\log w} K(x, w)\right) .
$$

When $L_{2}>x^{1 / 5}, x^{1 / 5}<w=o(x)$ and $1 \leq t \leq Y$, both sides of (2.4) are bounded and bounded away from zero.

Note. $L_{2}<x^{1 / 5}$ if and only if $\nu \geq 3 / 8$.

Proof. In the ranges preceding (2.4),

$$
t w^{4} f(w)<x, \quad t^{3 / 2} w f(w)<x
$$

and hence

$$
u(x / t, w) \geq 3, \quad \frac{\log t}{\log w} \leq \frac{2}{3} u(x, w) .
$$


Thus the conditions of Lemma 2.2(iii) hold with $u=u(x / t, w)$ in (a) and $u=u(x, w), v=\log t / \log w$ in (b). Since

it follows that

$$
\log u(x / t, w)=\log \frac{\log x}{\log w}+O(1)
$$

$$
\begin{aligned}
\varrho^{\prime}(u(x / t, w)) & =-K(x, w) \varrho(u(x / t, w)) \\
& =-K(x, w) \varrho(u(x, w)) \exp \left(\frac{\log t}{\log w} K(x, w)\right)
\end{aligned}
$$

as required.

Let

$$
I(w)=-\int_{1}^{Y} \frac{t-[t]}{t^{2} \log w} \varrho^{\prime}(u(x / t, w)) d t
$$

where $Y$ is given by (2.2), (2.3). By Lemma 2.2(ii), $I(w) \geq 0$. When $1 \ll I(w) \log w \ll 1$, we write $I(w) \asymp 1 / \log w$.

Lemma 2.4. For $Z \leq w \leq \min \left(L_{2}, x^{1 / 5}\right)$,

$$
I(w)=(C+o(1)) \frac{K(x, w)}{\log w} \varrho(u(x, w))
$$

where

$$
C=\int_{1}^{\infty} \frac{t-[t]}{t^{2}} d t
$$

When $L_{2}>x^{1 / 5}$ and $2<\log x / \log w \ll 1, I(w) \asymp 1 / \log w$.

Proof. Let $K_{1}(x, w)=K(x, w) / \log w, Y_{1}=\exp \left(\left(\log _{2} x\right)^{1 / 2}\right)$. For $w \geq Z$, $1 \leq t \leq Y_{1}$, we have $K_{1}(x, w) \log t=o(1)$. By Lemma 2.3 , when $Z \leq w \leq$ $\min \left(L_{2}, x^{1 / 5}\right)$,

$$
I(w)=\frac{K(x, w)}{\log w} \varrho(u(x, w)) \int_{1}^{Y} \frac{t-[t]}{t^{2-K_{1}(x, w)}} d t
$$

and this integral equals

$$
(1+o(1)) \int_{1}^{Y_{1}} \frac{t-[t]}{t^{2}} d t+O\left(\int_{Y_{1}}^{Y} \frac{1}{t^{2-K_{1}(x, w)}} d t\right)=C+o(1) .
$$

The last sentence of this lemma is a consequence of the last sentence of the previous lemma.

Note. When $\nu \geq 3 / 8$, we see that $L_{2}<x^{1 / 5}$ and $I(w)$ is increasing on $\left[Z, L_{2}\right]$. When $\nu<3 / 8, L_{2}=X>x^{1 / 5}$; by considering the integrand, we find that there exists a fixed $\gamma, 0<\gamma \leq 1 / 5$, such that $I(w)$ is increasing on $\left[Z, x^{\gamma}\right]$. 
LEMMA 2.5. Let $\varepsilon>0$. Uniformly for y satisfying

$$
\left(\log _{2} x\right)^{5 / 3+\varepsilon} \leq \log y \leq \log x \quad \text { and } \quad x \geq x_{0}(\varepsilon),
$$

we have

$$
\Psi(x, y)=x \varrho(u)\left(1+O\left(\frac{\log (u+1)}{\log y}\right)\right)
$$

where $u=\log x / \log y$ and $\Psi(x, y)$ is defined in (1.22).

For this range, the result is due to Hildebrand [4]. The error term is rather weak. Various other better approximations to $\Psi(x, y)$ have been developed. We employ one based on a function introduced by de Bruijn [1].

Definition. Let

$$
\Lambda(x, y)= \begin{cases}x \int_{0}^{\infty} \varrho\left(\frac{\log (x / t)}{\log y}\right) d\left(\frac{[t]}{t}\right) & \text { for } x \notin \mathbb{N}, \\ \frac{1}{2}(\Lambda(x-0, y)+\Lambda(x+0, y)) & \text { for } x \in \mathbb{N} .\end{cases}
$$

Lemma 2.6. Let $u=\log x / \log y$. For $x \notin \mathbb{N}$,

$$
\Lambda(x, y)=x\left\{\varrho(u)-\int_{1}^{x} \frac{t-[t]}{t^{2} \log y} \varrho^{\prime}\left(u-\frac{\log t}{\log y}\right) d t\right\} .
$$

Proof. Recall that $\varrho(u-\log t / \log y)=0$ for $t>x$ and $[t]=0$ for $0 \leq t<1$. Thus for $x \notin \mathbb{N}$,

$$
\Lambda(x, y)=x \int_{1-0}^{x+0} \varrho\left(u-\frac{\log t}{\log y}\right) d\left(\frac{[t]-t}{t}\right) .
$$

The result of the lemma is obtained by integrating by parts.

LEMma 2.7. Let $\varepsilon>0$. Uniformly for y satisfying (2.6)

$$
\Psi(x, y)=\Lambda(x, y)\left(1+O_{\varepsilon}\left(\exp \left(-(\log y)^{3 / 5-\varepsilon}\right)\right)\right) .
$$

This result is due to Saias [8]. De Bruijn proved in [1] that

$$
\Psi(x, y)=\Lambda(x, y)+O_{\varepsilon}\left(x \exp \left(-(\log y)^{3 / 5-\varepsilon}\right)\right)
$$

for $x>1, y \geq 2$; this agrees with Lemma 2.7 when $\log y \geq(\log x)^{5 / 8+\varepsilon}$, $x \geq x_{0}(\varepsilon)$.

Throughout this paper, $p$ denotes a prime.

Definition. For $\varepsilon>0$, write

$$
R(x, \varepsilon)=\exp \left(-(\log x)^{3 / 5-\varepsilon}\right) .
$$


Lemma 2.8. Let $\varepsilon>0$, with $\varepsilon<3 / 5-\nu$ if $\nu<3 / 5$, and let $h(w)$ be a positive increasing function with a continuous derivative on $w \geq 2$. Then

$$
S(x)=\sum_{p \leq x} \frac{1}{p h(p)}=J(x)+C+O\left(\frac{1}{h(x)} R(x, \varepsilon / 2)\right),
$$

where $C=C(h)$ is a constant given by (2.9) and

$$
J(x)=\int_{2}^{x} \frac{1}{w h(w) \log w} d w .
$$

Proof. We use the prime number theorem in the form

$$
\theta(x)=\sum_{p \leq x} \log p=x(1+E(x)) \quad \text { with } \quad E(x)=O_{\varepsilon}(R(x, \varepsilon / 2)) .
$$

By partial summation

$$
S(x)=\frac{E(x)}{h(x) \log x}+J(x)+C+\int_{x}^{\infty} w E(w) \frac{d}{d w}\left(\frac{1}{w h(w) \log w}\right) d w
$$

where

$$
C=\frac{1}{h(2) \log 2}-\int_{2}^{\infty} w E(w) \frac{d}{d w}\left(\frac{1}{w h(w) \log w}\right) d w .
$$

This infinite integral converges, as we now show.

For $z>x$,

$$
\begin{aligned}
\mid \int_{x}^{z} w E(w) \frac{d}{d w} & \left(\frac{1}{w h(w) \log w}\right) d w \mid \\
& \ll_{\varepsilon} \int_{x}^{z} w R(w, \varepsilon / 2) \frac{d}{d w}\left(\frac{-1}{w h(w) \log w}\right) d w=I_{1}(x, z), \quad \text { say. }
\end{aligned}
$$

Since

$$
\frac{\frac{d}{d w}(w R(w, \varepsilon / 2))}{R(w, \varepsilon / 2)}=1-\frac{3 / 5-\varepsilon / 2}{(\log w)^{2 / 5+\varepsilon / 2}},
$$

integration by parts yields

$$
\begin{aligned}
I_{1}(x, z) & =\left[\frac{-R(w, \varepsilon / 2)}{h(w) \log w}\right]_{x}^{z}+\int_{x}^{z} \frac{R(w, \varepsilon / 2)}{w h(w) \log w}\left(1+O\left((\log w)^{-2 / 5}\right)\right) d w \\
& \ll \frac{R(x, \varepsilon / 2)}{h(x)}+\frac{1}{h(x)} \int_{x}^{z} \frac{d}{d w}(-R(w, \varepsilon / 2)) d w \\
& \ll \frac{R(x, \varepsilon / 2)}{h(x)}
\end{aligned}
$$

as required. 
LEMMA 2.9. Let $g(w)$ be a positive increasing function with a continuous derivative on $w \geq 2$ and suppose that $h(w)=f(w)$ or $f(w) / R(w, \varepsilon / 2)$. Then for $x>v \geq 2$

$$
\begin{aligned}
\sum_{v<p \leq x} \frac{g(p)}{p h(p)}= & \int_{v}^{x} \frac{g(w)}{w h(w) \log w}(1+O(R(w, 3 \varepsilon / 4))) d w \\
& +O\left(\max _{w=v, x}\left(\frac{g(w)}{h(w)} R(w, \varepsilon / 2)\right)\right) .
\end{aligned}
$$

Proof. Write

$$
E_{1}(x)=S(x)-J(x)-C
$$

so $E_{1}(x) \ll R(x, \varepsilon / 2) / h(x)$ by Lemma 2.8 . By partial summation,

$$
\begin{aligned}
\sum_{v<p \leq x} \frac{g(p)}{p h(p)}= & {\left[E_{1}(w) g(w)\right]_{v}^{x} } \\
& +\int_{v}^{x} \frac{g(w)}{w h(w) \log w} d w-\int_{v}^{x} E_{1}(w) g^{\prime}(w) d w .
\end{aligned}
$$

For $n=1,2$ we have, using Lemma 2.1,

$$
\begin{aligned}
& \frac{\frac{d}{d w}\left(\frac{1}{f(w)} R^{n}(w, \varepsilon / 2)\right)}{\frac{1}{f(w)} R^{n}(w, \varepsilon / 2)} \\
& \quad=-\frac{1}{w \log w}\left((\nu+\xi(w)) \log f(w)+n\left(\frac{3}{5}-\frac{\varepsilon}{2}\right)(\log w)^{3 / 5-\varepsilon / 2}\right) .
\end{aligned}
$$

Hence

$$
\left|\int_{v}^{x} E_{1}(w) g^{\prime}(w) d w\right| \ll \int_{v}^{x} \frac{1}{h(w)} R(w, \varepsilon / 2) g^{\prime}(w) d w
$$

and the right side equals

$$
\begin{aligned}
{\left[\frac{1}{h(w)} R(w, \varepsilon / 2) g(w)\right]_{v}^{x} } & \\
& +\int_{v}^{x} \frac{g(w)}{w h(w) \log w} R(w, \varepsilon / 2) O\left(\log f(w)+(\log w)^{3 / 5-\varepsilon / 2}\right) d w \\
\ll & \max _{w=v, x}\left(\frac{g(w)}{h(w)} R(w, \varepsilon / 2)\right)+\int_{v}^{x} \frac{g(w)}{w h(w) \log w} R(w, 3 \varepsilon / 4) d w .
\end{aligned}
$$

The result of the lemma now follows. 
Lemma 2.10. Suppose that $\lim _{w \rightarrow \infty} \log f(w) / \log w=0$. Then

$$
\sum_{p>W}\left[\frac{x}{p f(p)}\right]=\int_{W}^{X} \frac{1}{\log w}\left[\frac{x}{w f(w)}\right] d w+O\left(\frac{x R(W, 3 \varepsilon / 4)}{f(W)}\right) .
$$

Proof. Recall that $W^{2} f(W)=x=X f(X)$ by (1.8), so for $p>X$,

$$
\left[\frac{x}{p f(p)}\right]=0 \text {. }
$$

For $1 \leq m<x /(W f(W))=W$, define $X(x / m)$ by

$$
X\left(\frac{x}{m}\right) f\left(X\left(\frac{x}{m}\right)\right)=\frac{x}{m},
$$

so $X=X(x)$. Then

$$
\sum_{p>W}\left[\frac{x}{p f(p)}\right]=\sum_{W<p \leq X} \sum_{m \leq x /(p f(p))} 1=\sum_{m<W} \sum_{W<p \leq X(x / m)} 1 .
$$

If we apply Lemma 2.9 with $h(w)=f(w), g(w)=w f(w)$ to the inner sum, the double sum becomes

$$
\begin{aligned}
\sum_{m<W}\left\{\int_{W}^{X(x / m)} \frac{1}{\log w}\right. & \left.(1+O(R(w, 3 \varepsilon / 4))) d w+O\left(\max _{w=W, X(x / m)}(w R(w, \varepsilon / 2))\right)\right\} \\
= & \int_{W}^{X} \frac{1}{\log w}(1+O(R(w, 3 \varepsilon / 4)))\left[\frac{x}{w f(w)}\right] d w \\
& +O\left(\sum_{m<W} X\left(\frac{x}{m}\right) R\left(X\left(\frac{x}{m}\right), \frac{\varepsilon}{2}\right)+W^{2} R(W, \varepsilon / 2)\right) .
\end{aligned}
$$

For $m<W$,

$$
W=X\left(\frac{x}{W}\right)<X\left(\frac{x}{m}\right)=\frac{x / m}{f(X(x / m))}<\frac{x}{m f(W)},
$$

and so

$$
\begin{aligned}
\sum_{m<W} X\left(\frac{x}{m}\right) R\left(X\left(\frac{x}{m}\right), \frac{\varepsilon}{2}\right) & <\frac{x}{f(W)} R(W, \varepsilon / 2) \sum_{m<W} \frac{1}{m} \\
& <\frac{x}{f(W)} R(W, 3 \varepsilon / 4) .
\end{aligned}
$$

Also

$$
\int_{W}^{X} \frac{R(w, 3 \varepsilon / 4)}{\log w}\left[\frac{x}{w f(w)}\right] d w<\frac{x R(W, 3 \varepsilon / 4)}{f(W)} \int_{W}^{X} \frac{1}{w \log w} d w \ll \frac{x R(W, 3 \varepsilon / 4)}{f(W)}
$$


since $\log _{2} X-\log _{2} W \sim \log 2$ when $\log f(w) / \log w \rightarrow 0$ as $w \rightarrow \infty$. On noting that $R(W, 3 \varepsilon / 4)>R(W, \varepsilon / 2)$ and $W^{2}=x / f(W)$, we obtain the result of the lemma.

3. Magnitude of $\Sigma(x)$. On writing $n=p m$ where $p=P(n)$, we see from the definition $(1.22)$ of $\Psi(x, y)$ that

$$
\begin{aligned}
\Sigma(x) & =\Sigma_{f}(x)=\sum_{\substack{n f(P(n)) \leq x \\
n>1}} 1=\sum_{p f(p) \leq x} \sum_{\substack{m \leq x /(p f(p)) \\
P(m) \leq p}} 1 \\
& =\sum_{p f(p) \leq x} \Psi\left(\frac{x}{p f(p)}, p\right) .
\end{aligned}
$$

Our first goal is to determine the dominant range for $p$ in the above sum and the magnitude of $\Sigma(x)$. We shall need to treat the cases $\nu>0, \nu=0$ separately. We begin by investigating the size of

$$
F(w)=\frac{1}{f(w)} \varrho(u(x, w))
$$

when $\nu>0$, where $u(x, w)$ is given by (1.16). Recall that $L=L(x)$ is given by (1.9). Define $\kappa$ by

$$
\kappa^{3}=\frac{\log _{3} x}{\log _{2} x}+|\xi(L)|+|\delta(L)|
$$

so $0<\kappa=o(1)$ by $(1.3)$ and (1.4).

Lemma 3.1. Let $\nu>0$. The maximum value of $F(w)$ occurs when $\log w \sim \log L$ and is of the form

$$
\exp \left(-\left(1+\nu+O\left(\kappa^{3}\right)\right) \log f(L)\right) .
$$

Proof. Consider first $F\left(L^{d}\right)$ when $\beta \leq d \leq \alpha$ where $\alpha$ satisfies (2.2) and

$$
\beta=\frac{2 \nu}{3(1+\nu)},
$$

so that $0<\beta<1<\alpha$; thus $d$ is bounded and its range includes 1. By (1.6),

$$
\log f\left(L^{d}\right)=\left(d^{\nu}+O(|\xi(L)|)\right) \log f(L) .
$$

We see from (1.16) that

$$
\begin{aligned}
u & =u\left(x, L^{d}\right)=\frac{\log x}{d \log L}\left(1-\frac{\log f\left(L^{d}\right)+d \log L}{\log x}\right) \\
& =\frac{\log x}{d \log L}\left(1+O\left(\frac{1}{\log _{2} x}\right)\right)
\end{aligned}
$$


by (1.11) and (1.12); we do not need the better error term that could be given here. It follows that

$$
\begin{aligned}
\log u+\log _{2} u & =\log _{2} x-\log _{2} L+\log _{3} x+O(1) \\
& =\frac{\nu+\delta(L)}{1+\nu+\delta(L)}\left(\log _{2} x+\log _{3} x\right)+O(1) .
\end{aligned}
$$

Hence by Lemma 2.2(i),

$$
\begin{aligned}
-\log \varrho(u) & =u\left(\log u+\log _{2} u+O(1)\right) \\
& =\frac{\log x \log _{2} x}{d \log L}\left(\frac{\nu}{1+\nu}+O\left(\frac{\log _{3} x}{\log _{2} x}+|\delta(L)|\right)\right) \\
& =\left(\frac{\nu}{d}+O\left(\frac{\log _{3} x}{\log _{2} x}+|\delta(L)|\right)\right) \log f(L) .
\end{aligned}
$$

Combining equations (3.2), (3.3), (3.6), (3.7) we obtain

$$
F\left(L^{d}\right)=\exp \left(-\left(d^{\nu}+\nu / d+O\left(\kappa^{3}\right)\right) \log f(L)\right) .
$$

The minimum value of $d^{\nu}+\nu / d$ occurs when $d=1$. Hence when $\beta \leq d \leq \alpha$, (3.4) is an upper bound of $F\left(L^{d}\right)$, and $F(L)$ is of the form (3.4).

It remains to show that $F(w)$ is of a smaller order of magnitude than (3.4) when $w<L^{\beta}$ or $w>L^{\alpha}$. As $w$ increases, $u(x, w)$ decreases and so $\varrho(u(x, w))$ increases. Hence for $w<L^{\beta}$,

$$
\begin{aligned}
F(w) & \ll \varrho(u(x, w)) \leq \varrho\left(u\left(x, L^{\beta}\right)\right)=\exp (-(\nu / \beta+o(1)) \log f(L)) \\
& =o(F(L))
\end{aligned}
$$

by (3.7) and since $\nu / \beta>1+\nu$. When $w>L^{\alpha}$,

$$
F(w) \ll \frac{1}{f(w)} \leq \frac{1}{f\left(L^{\alpha}\right)}=\exp \left(-\left(\alpha^{\nu}+o(1)\right) \log f(L)\right)=o(F(L))
$$

by (3.6) and since $\alpha^{\nu}>1+\nu$. The lemma now follows.

We remark that the derivative $F^{\prime}(w)$ has no sign changes in the intervals $L^{\beta} \leq w \leq L^{1-\kappa}, L^{1+\kappa} \leq w \leq L^{\alpha}$, and hence $F(w)$ has its maximum value for some $w$ in the range $L^{1-\kappa} \leq w \leq L^{1+\kappa}$. From (3.8) we deduce the

Corollary.

$$
F\left(L^{1 \pm \kappa}\right)=\exp \left(-\left(1+\nu+\frac{1}{2} \nu(\nu+1) \kappa^{2}+O\left(\kappa^{3}\right)\right) \log f(L)\right) .
$$

Proof. This follows since

$$
(1 \pm \kappa)^{\nu}+\frac{\nu}{1 \pm \kappa}=1+\nu+\frac{1}{2} \nu(\nu+1) \kappa^{2}+O\left(\kappa^{3}\right)
$$

We shall also need an estimate for the maximum value of

$$
G_{\varepsilon}(w)=F(w) R(w, \varepsilon)
$$


where $R(w, \varepsilon)$ is given by (2.7). We note that as $w \rightarrow \infty$,

$$
f(w) R(w, \varepsilon) \rightarrow \infty \quad \text { if } \nu \geq 3 / 5, \quad f(w) R(w, \varepsilon) \rightarrow 0 \quad \text { if } 0 \leq \nu<3 / 5 .
$$

Lemma 3.2. (i) When $\nu \geq 3 / 5$, the maximum value of $G_{\varepsilon}(w)$ occurs when $\log w \sim \log L$ and is of the form (3.4).

(ii) When $0 \leq \nu<3 / 5$, the maximum value of $G_{\varepsilon}(w)$ occurs when $\log w \sim \log M$ and is of the form

$$
\exp \left(-(1+o(1)) \frac{\log x \log _{2} x}{\log M}\right)
$$

where $M$ is given by (1.10).

Proof. (i) When $\nu \geq 3 / 5$, the required result follows from the proof of Lemma 3.1 since

$$
-\frac{\log R(w, \varepsilon)}{\log f(w)}=(\log w)^{3 / 5-\nu-\varepsilon-\delta(w)}=o\left(\frac{\log _{3} w}{\log _{2} w}\right)
$$

as $w \rightarrow \infty$; for $|\delta(w)|<\varepsilon / 2$ for large enough $w$.

(ii) When $0 \leq \nu<3 / 5$,

$$
-\frac{\log f(w)}{\log R(w, \varepsilon)}=o\left(\frac{\log _{3} w}{\log _{2} w}\right)
$$

so

$$
-\log G_{\varepsilon}(w)=\left(1+o\left(\frac{\log _{3} w}{\log _{2} w}\right)\right)(\log w)^{3 / 5-\varepsilon}-\log \varrho(u(x, w)) .
$$

We can now apply the method of Lemma 3.1 to this expression with $L$ replaced by $M$, taking the dominant interval to be of the form

$$
M^{\beta_{0}} \leq w \leq M^{\alpha_{0}} \quad \text { where } 0<\beta_{0}<\frac{3-5 \varepsilon}{8-5 \varepsilon}<1<\left(\frac{8}{5}-\varepsilon\right)^{5 /(3-5 \varepsilon)}<\alpha_{0} .
$$

The result then follows.

We defined $L_{2}$ in (2.2), (2.3). We now define $L_{1}$.

Definition. When $\nu \geq 3 / 5$, let

$$
L_{1}=L^{\beta}
$$

where $\beta$ is given by (3.5) and $L$ by (1.9). When $0 \leq \nu<3 / 5$, define $\beta_{1}$ and $L_{1}$ by

$$
\beta_{1}=\frac{3-5 \varepsilon}{8-5 \varepsilon}, \quad L_{1}=M^{\beta_{1}}
$$

where $M$ is given by (1.10) and we assume $0<\varepsilon<3 / 5$.

We observe that $0<\beta_{1}<3 / 8$ and so for $0<\nu<3 / 5$,

$$
L_{1}<M<L<L_{2}
$$

on using (1.14) and (2.3). 
Our next aim is to show that the dominant range in the sum (3.1) is $L_{1}<p \leq L_{2}$. Write

$$
\begin{aligned}
\Sigma(x) & =\left(\sum_{p \leq L_{1}}+\sum_{L_{1}<p \leq L_{2}}+\sum_{p>L_{2}}\right) \Psi\left(\frac{x}{p f(p)}, p\right) \\
& =\Sigma_{1}(x)+\Sigma_{2}(x)+\Sigma_{3}(x) .
\end{aligned}
$$

LEMMA 3.3. (i) When $\nu \geq 3 / 5$,

$$
\Sigma(x)-\Sigma_{2}(x) \ll x \exp \left(-\frac{3}{2}(1+\nu+o(1)) \log f(L)\right) .
$$

(ii) When $0 \leq \nu<3 / 5$,

$$
\Sigma(x)-\Sigma_{2}(x) \ll x \exp \left(-(1+o(1)) \frac{\log x \log _{2} x}{\log M}\right) .
$$

Proof. With $Z=Z(x)$ defined by (2.1), we find on using Lemma 2.5 that

$$
\begin{aligned}
\Sigma_{1}(x) & =\left(\sum_{p \leq Z}+\sum_{Z<p \leq L_{1}}\right) \Psi\left(\frac{x}{p f(p)}, p\right) \\
& \ll Z \Psi(x, Z)+x \sum_{Z<p \leq L_{1}} \frac{1}{p f(p)} \varrho(u(x, p)) \\
& \ll x Z \varrho\left(\frac{\log x}{\left(\log _{2} x\right)^{2}}\right)+x \varrho\left(u\left(x, L_{1}\right)\right) \sum_{Z<p \leq L_{1}} \frac{1}{p f(p)}
\end{aligned}
$$

since $u(x, p)$ decreases and so $\varrho(u(x, p))$ increases as $p$ increases. The sum on the right is bounded when $\nu>0$ and is $O\left(\log _{2} x\right)$ when $\nu=0$. From Lemma 2.2(i),

$$
\varrho\left(\frac{\log x}{\left(\log _{2} x\right)^{2}}\right)=\exp \left(-(1+o(1)) \frac{\log x}{\log _{2} x}\right) .
$$

When $\nu \geq 3 / 5, \varrho\left(u\left(x, L_{1}\right)\right)=\varrho\left(u\left(x, L^{\beta}\right)\right)$ is given by $(3.7)$ with $d=\beta$, where $\nu / \beta=\frac{3}{2}(1+\nu)$ by (3.5). When $0 \leq \nu<3 / 5$, we find similarly that

$$
\varrho\left(u\left(x, L_{1}\right)\right)=\varrho\left(u\left(x, M^{\beta_{1}}\right)\right)=\exp \left(-(1+o(1)) \frac{\log x \log _{2} x}{\log M}\right) .
$$

It follows that, in either case, $\Sigma_{1}(x)$ is bounded above by the expression on the right in the statement of the lemma.

When $p>X,[x /(p f(p))]=0$ so $\Psi(x /(p f(p)), p)=0$. Hence

$$
\Sigma_{3}(x)=\sum_{p>L_{2}} \Psi\left(\frac{x}{p f(p)}, p\right) \leq \sum_{L_{2}<p \leq X} \frac{x}{p f(p)} \ll \frac{1}{f\left(L_{2}\right)} x \log _{2} x .
$$


When $\nu \geq 3 / 5, L_{2}=L^{\alpha}$ where $\alpha^{\nu}=\frac{3}{2}(1+\nu)$ by (2.2); thus by (3.10), $\frac{1}{f\left(L_{2}\right)}=\exp \left(-\left(\alpha^{\nu}+o(1)\right) \log f(L)\right)=\exp \left(-\frac{3}{2}(1+\nu+o(1)) \log f(L)\right)$, giving the required bound for $\Sigma_{3}(x)$ in this case.

When $3 / 8 \leq \nu<3 / 5$,

$$
\log f\left(L_{2}\right)=\frac{\log x \log _{2} x}{\log M}
$$

by (2.3) and so $\Sigma_{3}(x)$ is bounded above by the expression on the right of (ii). When $0 \leq \nu<3 / 8, L_{2}=X$ and so $\Sigma_{3}(x)$ is empty.

When $\nu>0$, let

$$
\Sigma_{2}^{\prime}(x)=\sum_{L^{\beta}<p \leq L^{\alpha}} \Psi\left(\frac{x}{p f(p)}, p\right)
$$

so $\Sigma_{2}^{\prime}(x)=\Sigma_{2}(x)$ for $\nu \geq 3 / 5$.

Corollary. When $\nu>0$,

$$
\Sigma(x)-\Sigma_{2}^{\prime}(x) \ll x \exp \left(-\frac{3}{2}(1+\nu+o(1)) \log f(L)\right) .
$$

This follows from the argument giving Lemma 3.3(i), which is valid for any $\nu>0$. We note that when $0<\nu<3 / 5$, the error term in Lemma 3.3(ii) is stronger than that in the Corollary.

Lemma 3.4. Let $\nu>0$. Then

$$
\Sigma(x)=x \exp \left(-\left(1+\nu+o\left(\kappa^{3}\right)\right) \log f(L)\right) .
$$

Proof. We show that the right side is a lower bound for $\Sigma(x)$ and an upper bound for $\Sigma_{2}^{\prime}(x)$, and the result then follows from the Corollary to Lemma 3.3.

Since $f(p)$ is increasing,

$$
\begin{aligned}
\Sigma(x) & =\sum_{\substack{n f(P(n)) \leq x \\
n>1}} 1 \geq \sum_{\substack{n f(P(n)) \leq x \\
1<P(n) \leq L}} 1 \geq \sum_{\substack{n \leq x / f(L) \\
P(n) \leq L}} 1=\Psi\left(\frac{x}{f(L)}, L\right) \\
& =\frac{x}{f(L)} \varrho(u(x, L)+1)\left(1+O\left(\frac{\log _{2} x}{\log L}\right)\right) \\
& =x \exp \left(-\left(1+\nu+O\left(\kappa^{3}\right)\right) \log f(L)\right)
\end{aligned}
$$

on using Lemma 2.5, (3.7) with $d=1$ and (3.3). 
By Lemma 2.5 again,

$$
\begin{aligned}
\Sigma_{2}^{\prime}(x) & =\sum_{L^{\beta}<p \leq L^{\alpha}} \Psi\left(\frac{x}{p f(p)}, p\right) \\
& =x\left(1+O\left(\frac{\log _{2} x}{\log L}\right)\right) \sum_{L^{\beta}<p \leq L^{\alpha}} \frac{1}{p f(p)} \varrho(u(x, p)) \\
& \leq x(1+o(1))\left(\max _{L^{\beta}<p \leq L^{\alpha}} F(p)\right) \sum_{L^{\beta}<p \leq L^{\alpha}} \frac{1}{p} \\
& \leq x \exp \left(-\left(1+\nu+O\left(\kappa^{3}\right)\right) \log f(L)\right)
\end{aligned}
$$

by Lemma 3.1 and since the sum over $p$ is bounded.

LEMma 3.5. When $\nu>0$,

$$
\Sigma(x)=x\left(1+O\left(\frac{\log _{2} x}{\log L}\right)\right) \int_{L^{\beta}}^{L^{\alpha}} \frac{1}{w f(w) \log w} \varrho(u(x, w)) d w .
$$

Proof. By Lemmas 2.5 and 2.9,

$$
\begin{aligned}
\Sigma_{2}^{\prime}(x)= & x\left(1+O\left(\frac{\log _{2} x}{\log L}\right)\right) \sum_{L^{\beta}<p \leq L^{\alpha}} \frac{1}{p f(p)} \varrho(u(x, p)) \\
= & x\left(1+O\left(\frac{\log _{2} x}{\log L}\right)\right)\left\{\int_{L^{\beta}}^{L^{\alpha}} \frac{1}{w f(w) \log w} \varrho(u(x, w)) d w\right. \\
& \left.+O\left(\varrho\left(u\left(x, L^{\beta}\right)\right)+\frac{1}{f\left(L^{\alpha}\right)}\right)\right\} .
\end{aligned}
$$

The result now follows from Lemma 3.4, the Corollary to Lemma 3.3, and (3.9), (3.10) together with the definitions (3.5), (2.2) of $\beta, \alpha$, respectively.

Our aim in the next section is to improve Lemma 3.5 by using Lemma 2.7 instead of Lemma 2.5 and we shall see that the error term in Lemma 3.5 is best possible. We shall need the next result which follows from the last two lemmas.

LEMma 3.6. When $\nu \geq 3 / 5$,

$$
\int_{L^{\beta}}^{L^{\alpha}} \frac{1}{w \log w} G_{\varepsilon}(w) d w \ll R\left(L^{1-\kappa}, \varepsilon\right) \int_{L^{\beta}}^{L^{\alpha}} \frac{1}{w \log w} F(w) d w .
$$

Proof. By Lemmas 3.4 and 3.5,

$$
\int_{L^{\beta}}^{L^{\alpha}} \frac{1}{w \log w} F(w) d w=\exp \left(-\left(1+\nu+O\left(\kappa^{3}\right)\right) \log f(L)\right) .
$$


For $\nu \geq 3 / 5, \beta \leq d \leq \alpha$

$$
-\log R\left(L^{d}, \varepsilon\right)=(d \log L)^{3 / 5-\varepsilon}=O\left((\log L)^{-\varepsilon / 2} \log f(L)\right) .
$$

Thus, since $\kappa^{3} \gg(\log L)^{-\varepsilon / 2},(3.16)$ holds with $F$ replaced by $G_{\varepsilon}$ on the left. Moreover $G_{\varepsilon}\left(L^{1 \pm \kappa}\right)$ is equal to the right side of (3.11), and $G_{\varepsilon}(w)$ increases on $\left[L^{\beta}, L^{1-\kappa}\right]$ and decreases on $\left[L^{1+\kappa}, L^{\alpha}\right]$. Hence

$$
\begin{aligned}
\int_{L^{\beta}}^{L^{\alpha}} \frac{1}{w \log w} G_{\varepsilon}(w) d w & =\int_{L^{1-\kappa}}^{L^{1+\kappa}} \frac{1}{w \log w} G_{\varepsilon}(w) d w+O\left(G_{\varepsilon}\left(L^{1 \pm \kappa}\right)\right) \\
& \leq R\left(L^{1-\kappa}, \varepsilon\right) \int_{L^{1-\kappa}}^{L^{1+\kappa}} \frac{1}{w \log w} F(w) d w+O\left(G_{\varepsilon}\left(L^{1 \pm \kappa}\right)\right) \\
& =R\left(L^{1-\kappa}, \varepsilon\right) \int_{L^{\beta}}^{L^{\alpha}} \frac{1}{w \log w} F(w) d w+O\left(G_{\varepsilon}\left(L^{1 \pm \kappa}\right)\right) .
\end{aligned}
$$

The result now follows since for $\nu \geq 3 / 5$,

$$
\begin{aligned}
\exp \left(-\left(\frac { 1 } { 2 } \left(\nu(\nu+1) \kappa^{2}+\right.\right.\right. & \left.\left.\left.O\left(\kappa^{3}\right)\right) \log f(L)\right)\right) \\
& =o\left(R\left(L^{1-\kappa}\right)\right)=o\left(\exp \left(-((1-\kappa) \log L)^{3 / 5-\varepsilon}\right)\right) .
\end{aligned}
$$

Finally in this section, we discuss the order of magnitude of $\Sigma(x)$ when $\nu=0$, and in particular show that the expression on the right of Lemma 3.3(ii) is smaller than $\Sigma_{2}(x)$. When $\nu>0$, the main contribution to $\Sigma(x)$ comes from the primes near $L$. However when $\nu=0$, the larger primes contribute significantly to $\Sigma(x)$. When $\nu=0$ but $(\log f(w))\left(\log _{2} w\right)^{-2} \rightarrow \infty$ as $w \rightarrow \infty$, we can find a dominant range of primes excluding $X$ and argue in an analogous way to that used when $0<\nu<3 / 8$. This approach breaks down for smaller functions $f$, for then the range $W<p \leq X$ cannot be excluded. We restrict ourselves to establishing bounds for $\Sigma(x)$ valid whenever $\nu=0$.

Define $V=V(x)$ by

$$
\log V=\frac{\log x}{\max \left(\log _{2} x, \log f(X)\right)} .
$$

Lemma 3.7. When $\nu=0$,

$$
\begin{gathered}
\frac{x}{f(X)} \ll \Sigma(x) \ll \frac{x}{f(V)}, \\
\Sigma(x)=x \int_{V}^{X} \frac{1}{w f(w) \log w} \varrho(u(x, w))\left(1+O\left(\frac{\log ^{+} u(x, w)}{\log w}\right)\right) d w
\end{gathered}
$$

where $\log ^{+} u=\max (1, \log u)$. 
Proof. We consider first the range $V<p \leq X$. By Lemmas 2.5 and 2.9,

$$
\begin{aligned}
\sum_{V<p \leq W} & \Psi\left(\frac{x}{p f(p)}, p\right) \\
& =x \sum_{V<p \leq W} \frac{1}{p f(p)} \varrho(u(x, p))\left(1+O\left(\frac{\log u(x, p)}{\log p}\right)\right) \\
& =x \int_{V}^{W} \frac{1}{w f(w) \log w} \varrho(u(x, w))\left(1+O\left(\frac{\log u(x, w)}{\log w}\right)\right) d w
\end{aligned}
$$

since the error terms involving $R(w, 3 \varepsilon / 4)$ are negligible. By Lemma 2.10 and since

$$
\int_{W}^{X} \frac{1}{\log w} d w \ll \frac{X}{\log X} \ll \frac{x}{f(X) \log x},
$$

we have

$$
\begin{aligned}
& \sum_{W<p \leq X} \Psi\left(\frac{x}{p f(p)}, p\right) \\
= & \sum_{W<p \leq X}\left[\frac{x}{p f(p)}\right] \\
= & x \int_{W}^{X} \frac{1}{w f(w) \log w} d w+O\left(\frac{x}{f(W)} R(W, 3 \varepsilon / 4)\right)+O\left(\frac{x}{f(X) \log x}\right) .
\end{aligned}
$$

Note that $\varrho(u(x, w))=1$ for $W \leq w \leq X$. On substituting $t=u(x, w)+1$, we see that

$$
\int_{V}^{X} \frac{1}{w \log w} \varrho(u(x, w)) d w \sim \int_{1}^{\infty} \frac{1}{t} \varrho(t-1) d t=C,
$$

say, as $x \rightarrow \infty$, where $C>0$. Hence by (3.18) and (3.19),

$$
\frac{x}{f(X)} \ll \sum_{V<p \leq X} \Psi\left(\frac{x}{p f(p)}, p\right) \ll \frac{x}{f(V)} .
$$

To complete the proof of (i), we need to look at the corresponding sum over primes $p \leq V$. The error term in Lemma 3.3(ii) estimates the sum over $p \leq L_{1}$ to be

$$
\ll x \exp \left(-(\log x)^{1 / 4}\right)=o\left(\frac{x}{f(X) \log x}\right)
$$


on using (1.13). By Lemma 2.2(i),

$$
\begin{aligned}
-\log \varrho(u(x, V)) & =(1+o(1)) \frac{\log x}{\log V} \log \frac{\log x}{\log V} \\
& =(1+o(1)) \max \left(\log _{2} x, \log f(X)\right) \max \left(\log _{3} x, \log _{2} f(X)\right) \\
& =\Delta(x),
\end{aligned}
$$

say. Hence

$$
\begin{aligned}
\sum_{L_{1}<p \leq V} \Psi\left(\frac{x}{p f(p)}, p\right) & \ll x \sum_{L_{1}<p \leq V} \frac{1}{p f(p)} \varrho(u(x, p)) \\
& \ll x \varrho(u(x, V)) \log _{2} x \\
& \ll x \exp (-(1+o(1)) \Delta(x)) \\
& =o\left(\frac{x}{f(X) \log x}\right) .
\end{aligned}
$$

We obtain (i) from (3.21)-(3.23).

Using the substitution in (3.20),

$$
\begin{aligned}
\int_{V}^{X} \frac{1}{w f(w) \log w} \varrho(u(x, w)) \frac{\log ^{+} u(x, w)}{\log w} d w \\
\qquad \frac{1}{f(X)} \int_{1}^{\infty} \frac{1}{\log x} \varrho(t-1) d t \gg \frac{1}{f(X) \log x}
\end{aligned}
$$

since the integral converges. Hence the error term in (ii) exceeds the error terms in (3.19), (3.22), (3.23), and so (ii) follows from (3.18) and (3.19).

4. Proof of Theorem 1. In Lemma 3.5, we used Lemma 2.5 to express $\Sigma(x)$ in terms of an integral, but our error term was weak. We aim now to obtain a much better error term by using Lemma 2.7 instead. Lemma 3.3 suggests that we start by considering $\Sigma_{2}(x)$.

First we need some definitions. Recall that $L_{1}$ is given by (3.13), (3.14) and $L_{2}$ by $(2.2),(2.3)$. Write

$$
L_{3}=\min \left(L_{2}, W\right)= \begin{cases}L_{2} & \text { if } \nu \geq 3 / 8 \\ W & \text { if } 0 \leq \nu<3 / 8\end{cases}
$$

and let

$$
H_{1}(x)=\int_{L_{1}}^{L_{3}} \frac{1}{w f(w) \log w}(\varrho(u(x, w))+I(w)) d w
$$

where $I(w)$ is defined by $(2.5)$. When $0 \leq \nu<3 / 8$, so $L_{2}=X$ and $L_{3}=W$, 
let

$$
H_{2}(x)=\int_{W}^{X}\left[\frac{x}{w f(w)}\right] \frac{1}{\log w} d w
$$

LEMMA 4.1. Let $\varepsilon>0$ be sufficiently small but fixed.

(i) When $\nu \geq 3 / 5$,

$$
\Sigma_{2}(x)=x\left(1+O\left(R\left(L^{1-\kappa}, 3 \varepsilon / 4\right)\right)\right) H_{1}(x) .
$$

(ii) When $3 / 8 \leq \nu<3 / 5$,

$$
\Sigma_{2}(x)=x H_{1}(x)+O\left(x \exp \left(-(1+o(1)) \frac{\log x \log _{2} x}{\log M}\right)\right) .
$$

(iii) When $0 \leq \nu<3 / 8$,

$$
\Sigma_{2}(x)=x H_{1}(x)+H_{2}(x)+O\left(x \exp \left(-(1+o(1)) \frac{\log x \log _{2} x}{\log M}\right)\right) .
$$

Proof. Without loss of generality, we can assume that $x /(p f(p)) \notin \mathbb{N}$ for all primes $p$. By Lemmas 2.6, 2.7, with $I(p)$ defined by (2.5),

$$
\begin{aligned}
& \sum_{L_{1}<p \leq L_{3}} \Psi\left(\frac{x}{p f(p)}, p\right) \\
& =\sum_{L_{1}<p \leq L_{3}} \Lambda\left(\frac{x}{p f(p)}, p\right)(1+O(R(p, \varepsilon / 2))) \\
& =x \sum_{L_{1}<p \leq L_{3}} \frac{1}{p f(p)}(\varrho(u(x, p))+I(p))(1+O(R(p, \varepsilon / 2)))+E_{1}
\end{aligned}
$$

where, with $Y$ defined by (2.2) or (2.3),

$$
\begin{aligned}
\left|E_{1}\right| & =x(1+o(1)) \sum_{\substack{L_{1}<p \leq L_{3} \\
p^{2} f(p) \leq x / Y}} \frac{1}{p f(p)} \int_{Y}^{x /\left(p^{2} f(p)\right)} \frac{t-[t]}{t^{2} \log p}\left|\varrho^{\prime}(u(x / t, p))\right| d t \\
& \ll x \sum_{\substack{L_{1}<p \leq L_{3} \\
p^{2} f(p) \leq x / Y}} \frac{1}{p f(p) \log p} \int_{Y}^{\infty} \frac{d t}{t^{2}} \ll \frac{x}{Y}
\end{aligned}
$$

since $\sum_{p} 1 /(p \log p)$ converges; we recall that $\varrho^{\prime}(u(x / t, p))=0$ when $t>x /\left(p^{2} f(p)\right)$.

Our next step is to apply Lemma 2.9 to the sum on the right of (4.4). As $p$ increases, $u(x, p)$ decreases and therefore $\varrho(u(x, p))$ increases. From the remark after Lemma $2.4, I(p)$ increases on $\left[Z, L_{4}\right]$ where, for some fixed $\gamma$ with $0<\gamma \leq 1 / 5$, 


$$
L_{4}= \begin{cases}L_{2}=L_{3} & \text { if } \nu \geq 3 / 8 \\ x^{\gamma} & \text { if } 0 \leq \nu<3 / 8\end{cases}
$$

When $0 \leq \nu<3 / 8$, we note that $L_{4}=x^{\gamma}<W=L_{3}<X=L_{2}$ by (1.8), (2.3), (4.1); adapting the proof of Lemma 2.9 we find that

$$
\begin{aligned}
\sum_{L_{4}<p \leq L_{3}} \frac{1}{p f(p)} I(p)= & \int_{L_{4}}^{L_{3}} \frac{1}{w f(w) \log w} I(w)(1+O(R(w, 3 \varepsilon / 4))) d w \\
& +O\left(\max _{L_{4} \leq w \leq L_{3}}\left(\frac{I(w)}{f(w)} R(w, \varepsilon / 2)\right)\right) .
\end{aligned}
$$

For $x^{\gamma} \leq w \leq W, I(w) \asymp 1 / \log w$ and $\varrho(u(x, w)) \gg 1$; hence the last error term in (4.7) is

$$
\ll \max _{x^{\gamma} \leq w \leq W}\left(\frac{\varrho(u(x, w))}{f(w) \log w} R(w, \varepsilon / 2)\right) \ll \max _{x^{\gamma} \leq w \leq W} G_{\varepsilon / 2}(w)
$$

by (3.12).

Applying Lemma 2.9 three times we have

$$
\begin{aligned}
& \sum_{L_{1}<p \leq L_{3}} \frac{1}{p f(p)} \varrho(u(x, p))(1+O(R(p, \varepsilon / 2))) \\
& =\int_{L_{1}}^{L_{3}} \frac{1}{w f(w) \log w} \varrho(u(x, w))(1+O(R(w, 3 \varepsilon / 4))) d w \\
& \quad+O\left(\max _{w=L_{1}, L_{3}}\left(\frac{\varrho(u(x, w))}{f(w)} R(w, \varepsilon / 2)\right)\right)
\end{aligned}
$$

and

$$
\begin{aligned}
\sum_{L_{1}<p \leq L_{4}} \frac{1}{p f(p)} I(p)= & \int_{L_{1}}^{L_{4}} \frac{1}{w f(w) \log w} I(w)(1+O(R(w, 3 \varepsilon / 4))) d w \\
& +O\left(\max _{w=L_{1}, L_{4}}\left(\frac{I(w)}{f(w)} R(w, \varepsilon / 2)\right)\right)
\end{aligned}
$$

By Lemma 2.4,

$$
I(w)=o(\varrho(u(x, w))) \quad \text { for } L_{1}<w \leq L_{3} .
$$

From (4.7)-(4.10) we see that the sum on the right of (4.4) equals

$$
\begin{aligned}
& x \int_{L_{1}}^{L_{3}} \frac{1}{w f(w) \log w}(\varrho(u(x, w))+I(w))(1+O(R(w, 3 \varepsilon / 4))) d w \\
& \quad+O\left(x \max _{w=L_{1}, L_{3}}\left(\frac{\varrho(u(x, w))}{f(w)} R(w, \varepsilon / 2)\right)\right)+O\left(x \max _{L_{4} \leq w \leq L_{3}} G_{\varepsilon / 2}(w)\right)
\end{aligned}
$$


where the last error term appears only when $L_{4}<L_{3}$ (see (4.6)). It then follows from (4.4) and (4.5) that

$$
\begin{aligned}
& \sum_{L_{1}<p \leq L_{3}} \Psi\left(\frac{x}{p f(p)}, p\right) \\
& =x H_{1}(x)+O\left(x \int_{L_{1}}^{L_{3}} \frac{1}{w \log w} G_{3 \varepsilon / 4}(w) d w\right) \\
& \quad+O\left(x\left(\max _{w=L_{1}, L_{3}} G_{\varepsilon / 2}(w)+\max _{L_{4} \leq w \leq L_{3}} G_{\varepsilon / 2}(w)\right)\right)+O(x / Y) .
\end{aligned}
$$

In order to estimate these error terms, we consider separately the three ranges for $\nu$ specified in the lemma and also consider the range $L_{3}=W<$ $p \leq X$ in case (iii).

(i) Let $\nu \geq 3 / 5$. Then $L_{1}=L^{\beta}, L_{3}=L_{2}=L^{\alpha}$. By Lemma 3.6,

$$
\begin{aligned}
\int_{L^{\beta}}^{L^{\alpha}} \frac{1}{w \log w} G_{3 \varepsilon / 4}(w) d w & \ll R\left(L^{1-\kappa}, 3 \varepsilon / 4\right) \int_{L^{\beta}}^{L^{\alpha}} \frac{1}{w \log w} F(w) d w \\
& \ll R\left(L^{1-\kappa}, 3 \varepsilon / 4\right) H_{1}(x)
\end{aligned}
$$

since $I(w)=o(\varrho(u(x, w)))$. From the definitions (2.2), (3.5) of $\alpha, \beta, Y$ and (3.9), (3.10),

$$
\max _{w=L^{\beta}, L^{\alpha}} G_{\varepsilon / 2}(w) \ll \exp \left(-\frac{3}{2}(1+\nu+o(1)) \log f(L)\right)
$$

and $Y^{-1}$ is bounded above by the right side of (4.12). Moreover by Lemmas 3.1 and 3.3(i) and the definition (2.7) of $R\left(L^{1-\kappa}, 3 \varepsilon / 4\right)$, the right side of (4.12) is of a smaller order of magnitude than $R\left(L^{1-\kappa}, 3 \varepsilon / 4\right) H_{1}(x)$. Lemma 4.1(i) now follows.

(ii) Let $3 / 8 \leq \nu<3 / 5$. By (3.14) and (2.3), $L_{1}=M^{\beta_{1}}$ and $\log f\left(L_{2}\right)$ $=\left(\log x \log _{2} x\right) / \log M=\log Y$, so $L_{2}=o(W)$ and moreover $L_{1}<M$ $<L<L_{2}=L_{3}$ by (3.15). By Lemma 3.2(ii), all error terms in (4.11) are

$$
\ll x \log _{2} x \max _{L_{1} \leq w \leq L_{2}} G_{3 \varepsilon / 4}(w) \ll x \exp \left(-(1+o(1)) \frac{\log x \log _{2} x}{\log M}\right) .
$$

This establishes (ii) of the lemma.

(iii) Let $0 \leq \nu<3 / 8$. In this case $L_{1}=M^{\beta_{1}}$ but $L_{2}=X$, so $L_{3}=W$, and $\log Y=\left(\log x \log _{2} x\right) / \log M>\log f(X)$. The error terms in (4.11) satisfy the bounds of (ii). It remains to look at the sum 


$$
\begin{aligned}
\sum_{W<p \leq X} \Psi\left(\frac{x}{p f(p)}, p\right) & =\sum_{W<p \leq X}\left[\frac{x}{p f(p)}\right] \\
& =\int_{W}^{X} \frac{1}{\log w}\left[\frac{x}{w f(w)}\right] d w+O\left(x \frac{R(W, 3 \varepsilon / 4)}{f(W)}\right) \\
& =H_{2}(x)+O\left(x \exp \left(-(\log W)^{3 / 5-3 \varepsilon / 4}\right)\right)
\end{aligned}
$$

by Lemma 2.10. Since $\log W \sim \frac{1}{2} \log x$ when $\nu<1$, the last error term is smaller than

$$
x \exp \left(-\frac{\log x \log _{2} x}{\log M}\right) .
$$

This completes the proof of the lemma.

Proof of Theorem 1. First we observe that the sum $\Sigma(x)$ also satisfies the result of Lemma 4.1, for the error terms in Lemma 3.3 are bounded by the error terms in (4.11). We also note that in case (i), when $\nu \geq 3 / 5$ and $x$ is sufficiently large,

$$
R\left(L^{1-\kappa}, 3 \varepsilon / 4\right)=\exp \left(-((1-\kappa) \log L)^{3 / 5-3 \varepsilon / 4}\right) \ll R(L, \varepsilon) .
$$

Next we extend the ranges of integration in the definition (4.2) of $H_{1}(x)$. Arguing in a similar way to the proof of Lemma 3.3, we see that when $\nu \geq 3 / 8$

$$
\int_{2}^{x} \frac{1}{w f(w) \log w}(\varrho(u(x, w))+I(w)) d w-H_{1}(x)
$$

is bounded by the error term of that lemma. When $0 \leq \nu<3 / 8$, so $L_{3}=W$, we note that

$$
\int_{W}^{x} \frac{1}{w f(w) \log w}(\varrho(u(x, w))+I(w)) d w=\int_{W}^{X} \frac{1}{w f(w) \log w} d w
$$

since $\varrho(u(x, w))=1$ for $W \leq w \leq X, \varrho(u(x, w))=0$ for $w>X$, and $\varrho^{\prime}(u(x / t, w))=0$, so $I(w)=0$, for $w>W$. Moreover the difference between (4.13) and (4.14) is bounded by the error term in Lemma 3.3(ii).

Finally we replace $I(w)$ by the corresponding integral over the range $1 \leq t \leq x$. This is permissible since

$$
\left|\int_{Y}^{x} \frac{t-[t]}{t^{2}} \varrho^{\prime}(u(x / t, w)) d t\right| \ll \frac{1}{Y} \quad \text { and } \quad \int_{2}^{x} \frac{1}{w f(w)(\log w)^{2}} d w \ll 1
$$

and so we incur the error term $O(x / Y)$ of (4.5) again.

It now follows from (1.17), (1.18) and Lemma 3.3 that in the statement of Lemma 4.1 we can replace $\Sigma_{2}(x)$ by $\Sigma(x), H_{1}(x)$ by $H(x)$ and, when $0 \leq \nu<3 / 8, H_{2}(x)$ by $-H_{0}(x)$, which gives Theorem 1 . 
We can deduce using the method above that the error term in Lemma 3.5 when $\nu>0$ is best possible. Applying Lemma 2.7 instead of Lemma 2.5 in the argument of Lemma 3.5, we see that

$$
\begin{aligned}
\Sigma(x)= & x\left(1+O\left(R\left(L^{\beta}, \varepsilon\right)\right)\right) \int_{L^{\beta}}^{L^{\alpha}} \frac{1}{w f(w) \log w}(\varrho(u(x, w))+I(w)) d w \\
& +O\left(x \exp \left(-\frac{3}{2}(1+\nu+o(1)) \log f(L)\right)\right) .
\end{aligned}
$$

By Lemma 2.4, when $L^{\beta} \leq w \leq L^{\alpha}$, we have

$$
I(w) \asymp \frac{\log _{2} x}{\log L} \varrho(u(x, w)) .
$$

Hence we deduce

Corollary 1. When $\nu>0$,

$$
\begin{aligned}
\Sigma(x)-x \int_{L^{\beta}}^{L^{\alpha}} \frac{1}{w f(w) \log w} \varrho(u(x, w)) d w & \\
& \asymp x \frac{\log _{2} x}{\log L} \int_{L^{\beta}}^{L^{\alpha}} \frac{1}{w f(w) \log w} \varrho(u(x, w)) d w .
\end{aligned}
$$

Hence the error term in Lemma 3.5 is best possible.

We proceed in a similar way in the case $\nu=0$. When $u(x / t, w)$ is unbounded,

$$
-\varrho^{\prime}(u(x / t, w)) \sim \log (u(x / t, w)) \varrho(u(x / t, w))
$$

by Lemma 2.2(iii)(a), and otherwise both sides are $\asymp 1$. Moreover, for $1 \leq t \leq Y, V \leq w \leq W$, we have

$$
\log (u(x / t, w)) \sim \log _{2} x-\log _{2} w, \quad \frac{\log t}{\log w} \log u(x, w)=o(1)
$$

where $V$ is given by (3.17). Hence by Lemma 2.2(iii)(b),

$$
\varrho(u(x / t, w))=\varrho\left(u(x, w)-\frac{\log t}{\log w}\right) \sim \varrho(u(x, w))
$$

for $u(x, w)$ unbounded, and so

$$
I(w) \sim \varrho(u(x, w)) \frac{\log u(x, w)}{\log w} \int_{1}^{Y} \frac{t-[t]}{t^{2}} d t .
$$

Since $I(w) \asymp 1 / \log w$ when $u(x, w)$ is bounded, we obtain, on applying Lemma 2.7 to the left side of (3.18),

Corollary 2. When $\nu=0$, the error term in Lemma 3.7(ii) is best possible. 
To establish Theorem 3 (without assuming the Riemann Hypothesis), we treat the sum

$$
\Sigma^{\prime}(x)=\Sigma_{f}^{\prime}(x)=\sum_{1<n \leq x} \frac{1}{f(P(n))}=\sum_{p \leq x} \frac{1}{f(p)} \Psi\left(\frac{x}{p}, p\right)
$$

in an analogous way to the method applied to $\Sigma(x)$. The main changes are as follows: throughout we replace

$$
\begin{gathered}
\varrho(u(x, w))=\varrho\left(\frac{\log x-\log f(w)}{\log w}-1\right) \text { by } \varrho\left(\frac{\log x}{\log w}-1\right), \\
W \text { by } \sqrt{x}, \quad X \text { by } x,
\end{gathered}
$$

and when $0 \leq \nu<3 / 8$,

$$
H_{2}(x) \text { (given by (4.3)) by } \int_{\sqrt{x}}^{x}\left[\frac{x}{w}\right] \frac{1}{f(w) \log w} d w .
$$

After making the consequential modifications in the argument above, we establish this part of Theorem 3.

5. Proof of Theorem 2. Throughout this section we assume that the Riemann Hypothesis holds. Several of the lemmas above depend directly or indirectly on the prime number theorem which, under the Riemann Hypothesis, can be stated in the form

$$
\theta(x)=x\left(1+O\left(x^{-1 / 2}(\log x)^{2}\right)\right)
$$

(which replaces (2.8)). For convenience of exposition, we work with an error term of the shape $O\left(x^{-1 / 2+\varepsilon}\right)$ with $\varepsilon>0$.

Saias remarked $([8]$, p. 81$)$ that, under the Riemann Hypothesis, the result of Lemma 2.7 becomes:

Lemma $2.7(\mathrm{RH})$. Let $\varepsilon>0$. Uniformly for $x \geq x_{0}(\varepsilon)$ and $(\log x)^{2+\varepsilon} \leq$ $y \leq x$,

$$
\Psi(x, y)=\Lambda(x, y)\left(1+O_{\varepsilon}\left(y^{-1 / 2+\varepsilon} \log x\right)\right) .
$$

Let

$$
R(x, \varepsilon)=x^{-1 / 2+\varepsilon}
$$

we use this definition of $R(x, \varepsilon)$ throughout this section, instead of $(2.7)$. This affects Lemmas 2.8-2.10 in Section 2, and the definition (3.12) of $G_{\varepsilon}(w)$ as well as subsequent lemmas depending on the definition of $R(x, \varepsilon)$.

In order that the improved error terms are reflected in the final result, we replace the parameter $M$ by $N$ defined in (1.19), and increase the boundary values of $\nu$ that separate the three cases considered (see below). For convenience, we assume that, when $\nu=1 / 2, \eta(w) / \sqrt{\log _{2} w}$ is monotonic. 
Definitions. (i) When $\log f(w) / \log w \rightarrow \infty$ as $w \rightarrow \infty$, define

$$
L_{1}=L^{\beta}, \quad L_{2}=L^{\alpha}, \quad Y=f\left(L_{2}\right)
$$

with $L, \beta, \alpha$ defined by (1.9), (3.5), (2.2), respectively.

(ii) When $\lim _{w \rightarrow \infty} \log f(w) / \log w=k(0 \leq k<\infty)$, define $N=N(x)$ by (1.19) and

$$
L_{1}=N^{1 / 2}, \quad \log Y=\frac{\log x \log _{2} x}{\log N}, \quad f\left(L_{2}\right)=\min (Y, f(X))
$$

where $X$ is given by (1.8).

We observe that case (i) of this definition applies when $\nu>1$ or $\nu=1$ and $\eta(w) \rightarrow \infty$ as $w \rightarrow \infty$; case (ii) applies otherwise, and in particular whenever $\nu<1$ (so $k=0)$. Also in case (ii),

$f\left(L_{2}\right)=Y \quad$ if and only if $\quad \nu>\frac{1}{2}$ or $\nu=\frac{1}{2}$ and $\lim _{w \rightarrow \infty} \frac{\eta(w)}{\sqrt{\log _{2} w}} \geq \sqrt{1-2 \varepsilon}$, $f(W) \geq Y \quad$ if and only if $\quad \nu>\frac{1}{2}$ or $\nu=\frac{1}{2}$ and $\lim _{w \rightarrow \infty} \frac{\eta(w)}{\sqrt{\log _{2} w}} \geq \sqrt{2(1-2 \varepsilon)}$, and when $\nu=1 / 2$,

$$
W \leq L_{2} \leq X \quad \text { if and only if } \quad \sqrt{1-2 \varepsilon} \leq \lim _{w \rightarrow \infty} \frac{\eta(w)}{\sqrt{\log _{2} w}} \leq \sqrt{2(1-2 \varepsilon)},
$$

on using the monotonicity of $\eta(w) / \sqrt{\log _{2} w}$ when $\nu=1 / 2$.

In the proof, we do not need to make special provision for this latter situation; the corresponding inequality did not arise in the proof of Theorem 1 , for either $L_{2}<W$ or $L_{2}=X$ held.

In case (i) when $\nu=1$, so $\eta(w) \rightarrow \infty$ as $w \rightarrow \infty$, we modify the definition of $\kappa$ given in (3.3) to

$$
\kappa^{3}=\frac{\log _{3} x}{\log _{2} x}+|\xi(L)|+|\delta(L)|+\frac{1}{\eta(L)},
$$

which is required in proving the analogue of Lemmas 3.2(i) and 3.6; in all other cases, $\kappa$ is defined by (3.3) as before.

To establish Theorem 2, we modify the proofs in Sections 3 and 4 by replacing:

(a) $M$ by $N$, then defining $L_{1}, L_{2}, Y$ as above;

(b) the condition $\nu \geq 3 / 5$ by $\log f(w) / \log w \rightarrow \infty$ as $w \rightarrow \infty$, and $0 \leq \nu<3 / 5$ by $\lim _{w \rightarrow \infty} \log f(w) / \log w<\infty$;

(c) the condition $3 / 8 \leq \nu<3 / 5$ by $\lim _{w \rightarrow \infty} \log f(w) / \log w<\infty$ and $Y \leq f(W)$ 
(d) the condition $0 \leq \nu<3 / 8$ by $\lim _{w \rightarrow \infty} \log f(w) / \log w=0$ and $Y>f(W)$

(e) Lemma 2.7 by Lemma $2.7(\mathrm{RH})$ (absorbing the factor $\log x$ into the relevant error terms);

(f) (2.7) by (5.1) (the new definition of $R(x, \varepsilon)$ ).

We observe that, since $0<\kappa=o(1)$,

$$
R\left(L^{1-\kappa}, 3 \varepsilon / 4\right)=\left(L^{1-\kappa}\right)^{-1 / 2+3 \varepsilon / 4} \leq L^{-1 / 2+\varepsilon}=R(L, \varepsilon)
$$

for sufficiently large $x$. On implementing the modifications outlined above and making the minor consequential changes, we deduce Theorem 2.

Theorem 3 under the assumption of the Riemann Hypothesis is derived in a similar way.

\section{References}

[1] N. G. de Bruijn, On the number of positive integers $\leq x$ and free of prime factors $>y$, Indag. Math. 13 (1951), 50-60.

[2] - , The asymptotic behaviour of a function occurring in the theory of primes, J. Indian Math. Soc. (N.S.) 15 (1951), 25-32.

[3] P. Erdös, A. Ivić and C. Pomerance, On sums involving reciprocals of the largest prime factor of an integer, Glas. Mat. Ser. III 21 (41) (1986), 283-300.

[4] A. Hildebrand, On the number of positive integers $\leq x$ and free of prime factors $>y$, J. Number Theory 22 (1986), 289-307.

[5] A. Ivić, On some estimates involving the number of prime divisors of an integer, Acta Arith. 49 (1987), 21-33.

[6] - On sums involving reciprocals of the largest prime factor of an integer II, ibid. 71 (1995), 229-251.

[7] A. Ivić and C. Pomerance, Estimates for certain sums involving the largest prime factor of an integer, in: Topics in Classical Number Theory (Budapest, 1981), Colloq. Math. Soc. János Bolyai 34, North-Holland, Amsterdam, 1984, 769-789.

[8] E. Saias, Sur le nombre des entiers sans grand facteur premier, J. Number Theory 32 (1989), 78-99.

[9] E. J. Scourfield, On some sums involving the largest prime divisor of n, Acta Arith. 59 (1991), 339-363.

[10] - Comparison of two dissimilar sums involving the largest prime factor of an integer, in: Analytic Number Theory, Proc. of a Conference in Honor of H. Halberstam, Vol. 2, B. C. Berndt et al. (eds.), Birkhäuser, Boston, 1996, 723-735.

Department of Mathematics

Royal Holloway

University of London

Egham, Surrey TW20 0EX, England

E-mail: e.scourfield@rhul.ac.uk 